\title{
Correction
}

\section{Correction: Gaser and Schlaug, Brain Structures Differ between Musicians and Non-Musicians}

Some of the data presented in Figure 1 of "Brain Structures Differ between Musicians and Non-Musicians" by Christian Gaser and Gottfried Schlaug, which appeared on pages 9240-9245 of the October 8, 2003 issue, was reused by the authors from the previous publication in Gaser and Schlaug's (2003) "Gray matter differences between musicians and non-musicians." Ann N Y Acad Sci 999:514-517.

DOI: 10.1523/JNEUROSCI.3403-13.2013 